\title{
Density-Functional Theory Study of Half-Metallic Heterostructures: Interstitial Mn in Si
}

\author{
Hua Wu, ${ }^{1,2}$ Peter Kratzer, ${ }^{3}$ and Matthias Scheffler ${ }^{1}$ \\ ${ }^{1}$ Fritz-Haber-Institut der Max-Planck-Gesellschaft, Faradayweg 4-6, D-14195 Berlin, Germany \\ ${ }^{2}$ II. Physikalisches Institut, Universität zu Köln, Zülpicher Strasse 77, D-50937 Köln, Germany \\ ${ }^{3}$ Fachbereich Physik, Universität Duisburg-Essen, Lotharstrasse 1, D-47048 Duisburg, Germany
}

(Received 15 November 2006; published 12 March 2007)

\begin{abstract}
Using density-functional theory within the generalized gradient approximation, we show that Si-based heterostructures with 1/4 layer $\delta$ doping of interstitial $\mathrm{Mn}\left(\mathrm{Mn}_{\mathrm{int}}\right)$ are half-metallic. For $\mathrm{Mn}_{\mathrm{int}}$ concentrations of $1 / 2$ or 1 layer, the states induced in the band gap of $\delta$-doped heterostructures still display high spin polarization, about $85 \%$ and $60 \%$, respectively. The proposed heterostructures are more stable than previously assumed $\delta$ layers of substitutional Mn. Contrary to widespread belief, the present study demonstrates that interstitial Mn can be utilized to tune the magnetic properties of $\mathrm{Si}$, and thus provides a new clue for Si-based spintronics materials.
\end{abstract}

PACS numbers: 75.50.Pp, 71.15.Mb, 73.20.At, 75.70.-i

Doping of semiconductors with magnetic $3 d$ transition metal impurities has attracted much interest: recent advances in spintronics have been largely due to magnetic semiconductors produced in this way. Their spin-dependent electric transport properties [1] could be exploited in novel magnetoelectronic devices, e.g., spin filters. Since it has long been known that manganese impurities can have a high magnetic moment [2,3], research has focused Mndoped magnetic semiconductors based on GaAs and $\mathrm{Ge}$ [4-6]. Moreover, doping of GaAs by a $\delta$ layer of Mn has been proposed theoretically [7], and an experimental realization has been reported recently [8]. The calculations of spin-polarized transport [7] employing density-functional theory (DFT) predicted that GaAs doped with a $\delta$ layer of substitutional $\mathrm{Mn}\left(\mathrm{Mn}_{\text {sub }}\right)$ on Ga sites is a two-dimensional (2D) ferromagnetic half-metal with large exchange coupling. Indeed, a Curie temperature of up to $250 \mathrm{~K}$ has been measured on $\mathrm{Mn} \delta$-doped GaAs samples [8]. Adding magnetic functionality to the most common semiconductor, $\mathrm{Si}$, is still in its infancy, despite recent reports about ferromagnetism in Mn-doped Si created by ion implantation [9]. Recently, Qian et al. [10] proposed, on the basis of pseudopotential calculations within the generalized gradient approximation (GGA), that a $\mathrm{Si}$ heterostructure with $\mathrm{Mn}_{\text {sub }} \delta$ doping should be a $2 \mathrm{D}$ ferromagnetic halfmetal. So far, research on $\mathrm{Mn}$-doped Si has concentrated on $\mathrm{Mn}_{\text {sub }}[11,12]$, probably motivated by the physics of dilute magnetic semiconductors, where it has been argued that $\mathrm{Mn}_{\text {sub }}$ is crucial for ferromagnetism [5,6,13,14]. However, these $\mathrm{Mn}_{\text {sub }}$ impurities in Si are energetically less stable than interstitial ones [15-17]. This has been considered a serious obstacle in creating magnetic $\mathrm{Mn}: \mathrm{Si}$ calling for growth techniques far from equilibrium [15].

In this Letter, we investigate the role of interstitial $\mathrm{Mn}$ $\left(\mathrm{Mn}_{\mathrm{int}}\right)$ impurities for ferromagnetism in Si by means of all-electron full-potential DFT calculations. We propose a novel type of heterostructures with $\mathrm{Mn}_{\text {int }} \delta$ doping. For occupation of each fourth interstitial site in a single layer by Mn, we find 2D half-metallic behavior with a KohnSham gap of $0.5 \mathrm{eV}$ in the electronic density of states in the majority spin channel. Even for higher doping densities, the spin polarization is still large, $>60 \%$, judged from the density of states at the Fermi level. This is different from the previously proposed $\mathrm{Mn}_{\text {sub }} \delta$ layers [10], where a (smaller) gap in the minority spin channel was responsible for the half-metallicity. Moreover, our proposed $\mathrm{Mn}_{\text {int }} \delta$ layer is calculated to be $0.5 \mathrm{eV}$ per $\mathrm{Mn}$ atom lower in energy than the heterostructure proposed by Qian et al. [10], in line with the known higher stability of isolated $\mathrm{Mn}_{\text {int }}$ impurities compared to $\mathrm{Mn}_{\text {sub }}$ impurities in $\mathrm{Si}$ $[16,17]$. Consequently, Si self-interstitials can destroy the half-metallicity of the heterostructure assumed by Qian et al. by energetically favorable site exchange with $\mathrm{Mn}_{\text {sub }}$.

Our proposed Si-based heterostructures are modeled by a Si(001) supercell with 16 atomic layers (see Fig. 1). Such thickness ensures that the central Si layer behaves as in the bulk, and the interaction between Mn layers is negligible. Mn deposited on a $\mathrm{Si}(001)$ surface occupies preferably subsurface interstitial sites [18], and a $c(4 \times 2)$ arrangement for $1 / 4$ monolayer (ML) $\mathrm{Mn}_{\text {int }}$ has been identified recently by a combined scanning tunneling microscopy (STM) and DFT study [19]. Therefore we first study the $c(4 \times 2)$ heterostructure consisting of 16-layer $\mathrm{Si}$ and $1 / 4 \mathrm{ML} \mathrm{Mn}_{\text {int }}$ in $c(4 \times 2)$ arrangement, as seen in Figs. 1(a) and 1(b). We also perform calculations for $1 / 4 \mathrm{ML} \mathrm{Mn}_{\text {int }}$ in $p(2 \times 2)$ arrangement (not shown). Secondly, we deal with a $p(2 \times 2)$ heterostructure with $1 / 2 \mathrm{ML} \mathrm{Mn}_{\text {int }}$, as shown in Fig. 1(c). Finally we compare the $(1 \times 1)$ heterostructure having $1 \mathrm{ML} \mathrm{Mn}_{\text {int }}$ [Figs. 1(d) and $1(\mathrm{e})]$ with the $(1 \times 1)$ heterostructure having $1 \mathrm{ML}$ $\mathrm{Mn}_{\text {sub }}$ [Figs. 1(f) and 1(g)] studied by Qian et al. [10].

We perform DFT-GGA [20] calculations using the fullpotential augmented plane-wave plus local-orbital method [21], which was shown to be appropriate for description of the Mn:Si system [18]. The lattice constant of $5.48 \AA$ for bulk Si calculated in GGA is used to construct the hetero- 
(a)

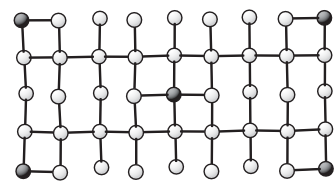

(b)

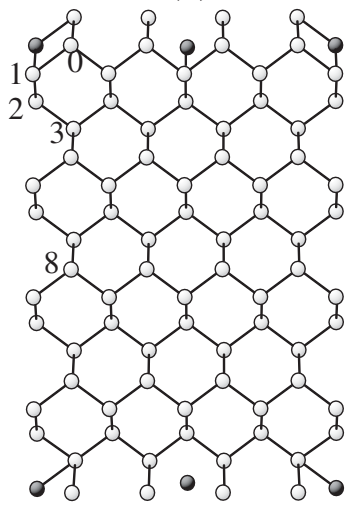

(c)

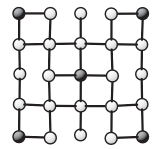

(d)

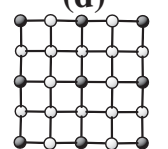

(e)

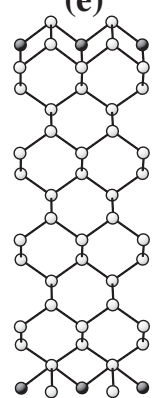

(f)

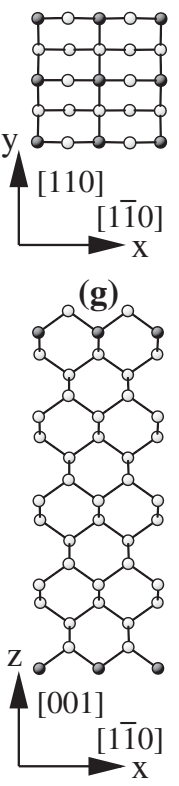

FIG. 1. (a) (001)-xy-plane view and (b) (110)-xz-plane view of the Si-based heterostructure (modeled by a 16-layer supercell) with $1 / 4$ layer of interstitial $\mathrm{Mn}$ in $c(4 \times 2)$ arrangement; (c) (001)-plane view of the heterostructure with $1 / 2$ layer of interstitial $\mathrm{Mn}$ in $p(2 \times 2)$ arrangement; (d) (001)-plane view and (e) (110)-plane view of the heterostructure with a full layer of interstitial $\mathrm{Mn}$ in $(1 \times 1)$ arrangement; (f) (001)-plane view and $(\mathrm{g})(110)$-plane view of the heterostructure with a full layer of substitutional $\mathrm{Mn}$ in $(1 \times 1)$ arrangement. Black circles show $\mathrm{Mn}$ and gray circles $\mathrm{Si}$ atoms. The silicon atoms in different layers are marked with indices.

structures we study. The muffin-tin sphere radii are chosen to be $1.11 \AA$ for both the $\mathrm{Mn}$ and $\mathrm{Si}$ atoms. Converged results are obtained at a cut-off energy of $13.8 \mathrm{Ry}$ for the interstitial plane-wave expansion, and with a set of $12 \times$ $12 \times 1$ special $\mathbf{k}$ points for integrations over the Brillouin zone of the $(1 \times 1)$ heterostructure (and an equivalent density of $\mathbf{k}$ points for larger supercells, e.g., $6 \times 5 \times 1$ for the $c(4 \times 2)$ cell). All Si and Mn atoms except for the central $\mathrm{Si}$ layer are relaxed until the calculated atomic force for each of them is smaller than $0.05 \mathrm{eV} / \AA$.

The $c(4 \times 2)$ heterostructure [see Figs. 1(a) and 1(b)] displays a half-metallic electronic band structure as shown by the density of states (DOS) derived from the KohnSham energy levels [see Fig. 2(a)]. The spin-up channel (dashed blue lines) is insulating with a Kohn-Sham gap of $0.5 \mathrm{eV}$. The spin-down channel (solid red lines) is metallic, and the Fermi level lies $0.2 \mathrm{eV}$ below the bottom of the spin-up conduction bands. While the Mn $3 d$ spin-up orbitals are fully occupied, the planar spin-down $x^{2}-y^{2}$ orbitals form a conduction band with bandwidth of $0.7 \mathrm{eV}$, contributing to the DOS in the metallic channel. The narrower $x z$ - and $y z$-derived conduction bands are also partially occupied and add to the DOS at the Fermi energy [22]. The coplanar $\mathrm{Si}\left(\mathrm{Si}_{0}\right)$, the first-neighbor-layer $\mathrm{Si}\left(\mathrm{Si}_{1}\right)$ and the second-neighbor-layer $\mathrm{Si}\left(\mathrm{Si}_{2}\right)$ [cf. Fig. 1(b)] also
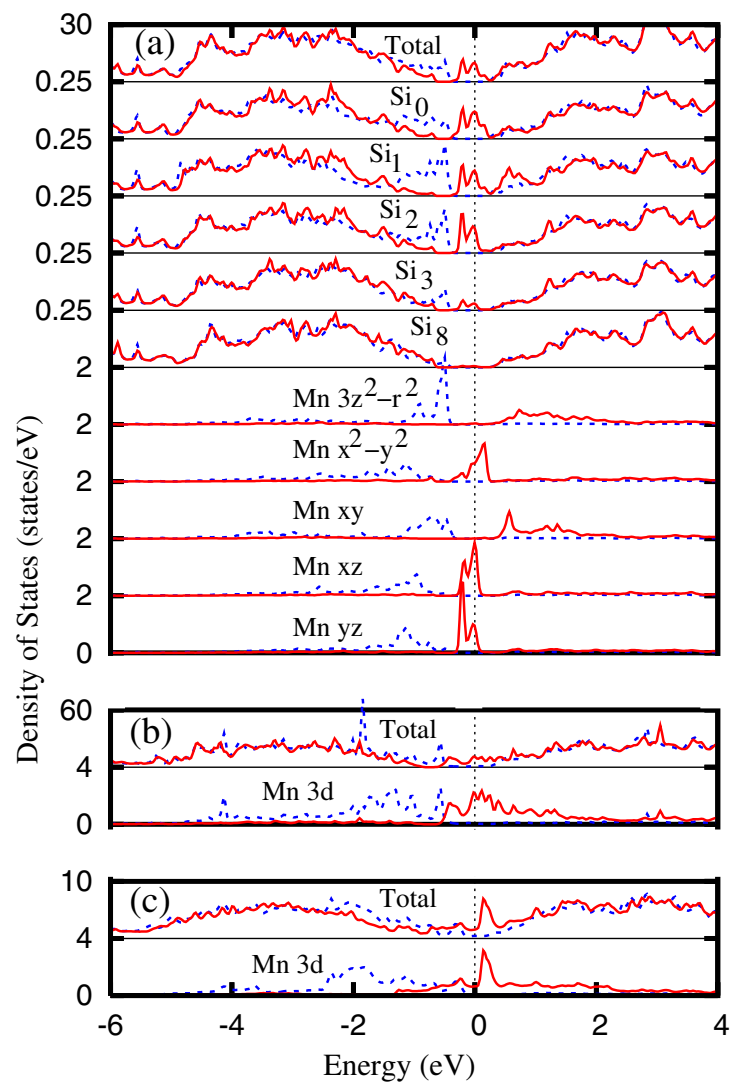

FIG. 2 (color online). DOS of (a) the 1/4-layer interstitial Mn $c(4 \times 2)$, (b) the 1/2-layer Mn $p(2 \times 2)$, and (c) the 1-layer Mn $(1 \times 1)$ heterostructures [cf. Figs. 1(a)-1(e)]. The solid red (dashed blue) line refers to the down (up) spin component. The Fermi level is set to zero. (a) shows half-metallicity, (b) and (c) a high spin polarization at the Fermi level.

contribute considerably to the half-metallic DOS through Mn $3 d-$ Si $3 s 3 p$ hybridizations. Such contribution decreases strongly for the third-neighbor-layer $\mathrm{Si}\left(\mathrm{Si}_{3}\right)$ and eventually vanishes for the central-layer $\mathrm{Si}\left(\mathrm{Si}_{8}\right)$. The $\mathrm{Mn}$ atom has a local spin magnetic moment (within the muffintin sphere) of $2.56 \mu_{B}$, and its four $\mathrm{Si}$ neighbors $\mathrm{Si}_{1}$ (to which it is tetrahedrally coordinated) get spin polarized with a spin moment of about $0.024 \mu_{B}$ each. Any other Si atom has a spin moment less than $0.01 \mu_{B}$. Taking also into account the spin moment of $0.25 \mu_{B}$ outside the muffin-tin spheres, stemming from the Mn $3 d-\mathrm{Si} 3 s 3 p$ hybridized bands, the total spin moment is an integer of $3 \mu_{B}$ per supercell [23]. Note that due to the long distance of $8.7 \AA$ between the center and corner $\mathrm{Mn}$ atom(s) in Fig. 1(a) the magnetic coupling turns out to be weak, but ferromagnetic (FM): Our calculations show that the FM state is slightly more stable than the antiferromagnetic (AFM) one by $5 \mathrm{meV} / \mathrm{Mn}$, consistent with the short range of FM interactions in zinc blende $\mathrm{MnSi}$ and $\mathrm{MnGe}[25,26]$.

The $1 / 4$ ML $p(2 \times 2)$ heterostructure (not shown, but refer to Fig. 1(c) and remove the central Mn in the $p(2 \times 2)$ cell) also displays half-metallicity, similar to the above discussed 1/4 ML $c(4 \times 2)$ heterostructure. The spindown $x^{2}-y^{2}$ orbital hybridized with Si $s p$ and the spin- 
down $x z$ and $y z$ orbitals determine the half-metallic DOS (not shown). The $p(2 \times 2)$ superstructure is only sightly less stable than the above $c(4 \times 2)$ one by $20 \mathrm{meV}$ per supercell, which shows that the $c(4 \times 2)$ superstructure gains a little more by lattice relaxation.

For the $1 / 2 \mathrm{ML} p(2 \times 2)$ heterostructure [see Fig. 1(c)], the DOS is displayed in Fig. 2(b), indicating a high spin polarization at the Fermi level of $85 \%$. The Mn spin moment is $2.72 \mu_{B}$ and the total one is $6.34 \mu_{B}$ per supercell $\left(3.17 \mu_{B}\right.$ per $\left.\mathrm{Mn}\right)$. Compared with the $c(4 \times 2)$ supercell, the conduction bands become broader due to decreased Mn-Mn distance and enhanced Mn $d-$ Si $s p$ hybridization. The FM exchange coupling of Mn to its four first neighbors at $5.5 \AA$ is quite large, $J=13 \mathrm{meV}$. This estimate is based on a Heisenberg model with local Mn spin of $S=3 / 2$, and the calculated stability of the FM state over the AFM state $4 J S^{2}=120 \mathrm{meV}$ per Mn atom. Thus the $1 / 2 \mathrm{ML} p(2 \times 2)$ heterostructure combines the advantage of a stronger inplane FM coupling with a still high spin polarization.

Now we turn to the $1 \mathrm{ML}(1 \times 1)$ heterostructure [see Figs. 1(d) and 1(e)]. Even in this case, spin-polarization is still $>60 \%$, as shown by our calculations [Fig. 2(c)]. The $\mathrm{Mn}$ and $\mathrm{Si}$ atoms form broad conduction bands. The $\mathrm{Mn}$ spin moment is $2.68 \mu_{B}$ and the total one is $3.12 \mu_{B}$ per $(1 \times 1)$ supercell. According to our calculations, the MnMn in-plane FM coupling turns out to be further increased: the FM state is more stable than the AFM state by $570 \mathrm{meV} / \mathrm{Mn}$. Since the direct magnetic coupling between two neighboring $\mathrm{Mn}$ atoms (with distance of $3.9 \AA$ ) is AFM, we attribute the increased FM coupling with shorter Mn-Mn distance to an enhanced interaction of doubleexchange type. It operates in the impurity band formed

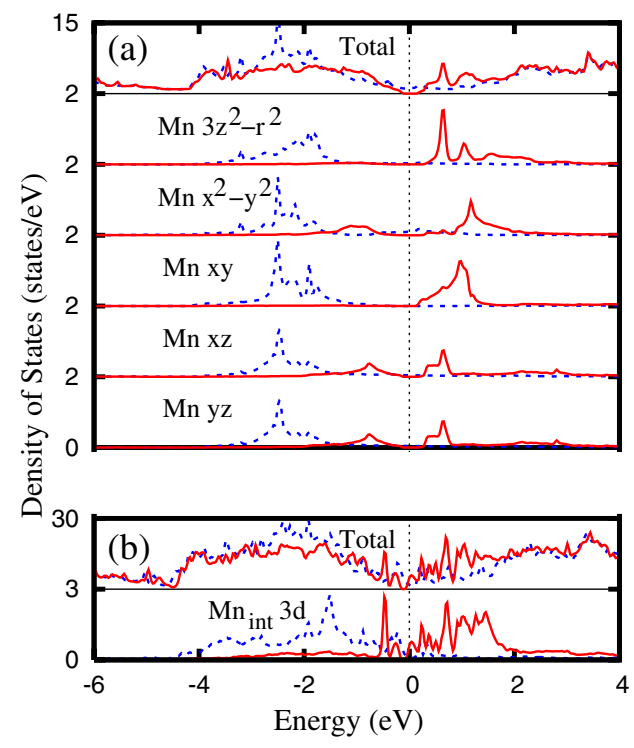

FIG. 3 (color online). DOS of the 1-layer substitutional Mn. (a) $(1 \times 1)$ heterostructure shows half-metallicity; (b) $p(2 \times 2)$ heterostructure with extra 1/4-layer coplanar interstitial $\mathrm{Si}$ shows, after an energetically favorable $\mathrm{Si}-\mathrm{Mn}$ site interchange, the loss of half-metallicity. The solid red (dashed blue) line refers to the down (up) spin component. by the spin-down $3 d$ states of Mn with $t_{2}$ character, as seen in Fig. 2, and is mediated by spin-down Si $s p$ states. As a result, we find the $\mathrm{Si}$ atoms to have small spin moments parallel to $\mathrm{Mn}$ in all the above calculations.

Next we show that the heterostructure with $\mathrm{Mn}_{\text {sub }}$ [Figs. 1(f) and 1(g)] proposed by Qian et al. [10] is energetically unfavorable. Figure 3(a) shows that our calculations yield half-metallic behavior with a Kohn-Sham gap of $0.25 \mathrm{eV}$ for $1 \mathrm{ML} \mathrm{Mn}_{\text {sub }}(1 \times 1)$, in agreement with Qian et al. [10]. The Mn spin moment is $2.97 \mu_{B}$, each firstneighbor Si has a spin moment of $-0.116 \mu_{B}$, and the total one is again integer, $3 \mu_{B}$ per $(1 \times 1)$ supercell. However, the $\mathrm{Mn}_{\text {sub }}$ heterostructure turns out to be less stable than the $\mathrm{Mn}_{\text {int }}$ one by $490 \mathrm{meV}$ per $(1 \times 1)$ supercell, assuming bulk $\mathrm{Si}$ as reservoir for the extra Si. Knowledge about MnSi compounds corroborates the metastability of tetrahedrally coordinated $\mathrm{Mn}_{\text {sub }}$ : $\mathrm{Mn}$ is known to prefer high coordination to $\mathrm{Si}$, as exemplified by the sevenfold $\mathrm{Mn}$ $\mathrm{Si}$ coordination in the ground-state crystal structure of $\mathrm{MnSi}$, or the eightfold coordination in the $\mathrm{CsCl}$ structure [18]. In contrast, the hypothetic zinc-blende structure of $\mathrm{MnSi}$ with tetrahedral coordination of $\mathrm{Mn}$ to $\mathrm{Si}$ is calculated to be extremely unstable, $2.3 \mathrm{eV}$ higher in energy than the ground state. The heterostructures with $\mathrm{Mn}_{\text {int }}$ proposed by us, having four second-neighbor $\mathrm{Si}$ atoms $(2.7 \AA)$ in addition to four first-neighbor Si atoms $(2.4 \AA)$, comply with the rules for the optimal Mn-Si coordination derivable from bulk phase stability.

We note that both types of $\delta$ layers considered are only metastable in the thermodynamic sense, i.e., they could be destroyed by annealing. However, the tendency of Mn to form ordered subsurface layers, as observed recently [19], suggests that growth kinetics could be exploited to fabricate the $\mathrm{Mn}_{\text {int }}$ heterostructure proposed by us. Although the reported $\mathrm{Mn}$-induced $c(4 \times 2)$ structure has so far been observed only in islands, extended layers might be obtainable by improved growth techniques, and could be overgrown at lower temperatures with a capping layer. After successful preparation, the heterostructures are still jeopardized by degradation due to highly mobile [27] Si selfinterstitials: By studying the $1 \mathrm{ML} \mathrm{Mn}_{\text {sub }} p(2 \times 2)$ heterostructure with an extra 1/4 ML coplanar interstitial Si, we find that interstitial $\mathrm{Si}$ is capable of site exchange with one $\mathrm{Mn}_{\text {sub }}$, thereby considerably lowering its energy by $1.2 \mathrm{eV}$. This irreversibly destroys the half-metallicity of the $\mathrm{Mn}_{\text {sub }}$ heterostructure (cf. Ref. [10]) by degrading the spin polarization at the Fermi level to about 40\%, as seen in Fig. 3(b). In the $\mathrm{Mn}_{\text {int }}$ heterostructure, trapped coplanar Si interstitials have a less drastic effect on the electronic structure, but they could shift the Fermi level out of the half-metallic gap if present in high concentrations.

We stress that the half-metallicity in our proposed $\mathrm{Mn}_{\text {int }}$ heterostructure (metallic minority spin channel) and in the $\mathrm{Mn}_{\text {sub }}$ heterostructure of Qian et al. (metallic majority spin channel) have different physical origin. This is explained in the level diagram of Fig. 4 . The $\mathrm{Mn}_{\text {int }} 3 d$ states are energetically located near the top of the Si-host valence bands 


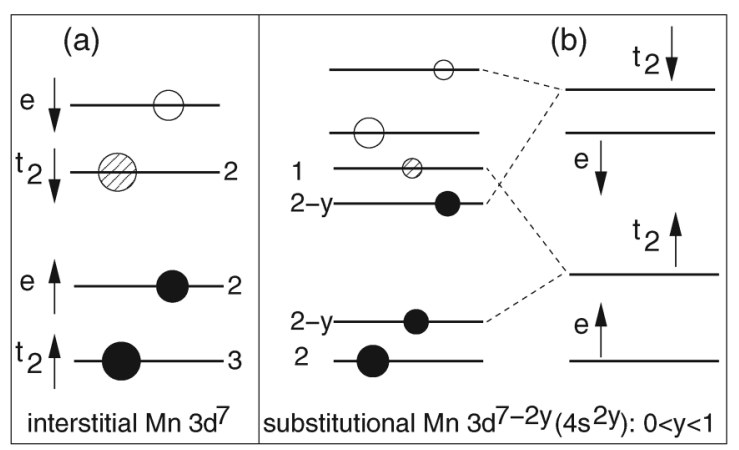

FIG. 4. Schematic view of the electronic states of the (a) interstitial and (b) substitutional $\mathrm{Mn}$ in Si [22]. The filled, patterned, and open circles indicate full, partial, and zero occupation, respectively. Both the size of the circles and the numbers next to the levels indicate their occupation number. The halfmetallic DOS results in (a) from the $t_{2 \downarrow}$ band, but in (b) from the $t_{2 \uparrow}$ antibonding band.

[Fig. 2(a)]. The weak crystal field with $T_{d}$ symmetry and strong Hund exchange stabilize a high-spin state for $\mathrm{Mn}_{\text {int }}$ $[2,3]$. The $4 s \rightarrow 3 d$ electron transfer leads to a $3 d^{7}$ $\left(t_{2 \uparrow}^{3} e_{\uparrow}^{2} t_{2 \downarrow}^{2}\right)$ configuration [Fig. 4(a)]. Thus the partially filled $t_{2 \downarrow}$ band is responsible for the metallic spin-down DOS seen in Fig. 2(a). For $\mathrm{Mn}_{\text {sub }}$, however, the Mn states of $t_{2}$ symmetry mix strongly with $\mathrm{Si} s p$ states of the same symmetry, thus splitting the $t_{2}$ states into a bonding and an antibonding band, lying in the valence and in the conduction band of the $\mathrm{Si}$ host, respectively [cf Fig. 3(a)]. The $\mathrm{Mn}_{\text {sub }} 3 d t_{2}^{4-2 y}$ and $4 s^{2 y}$, in total four electrons, fill up the bonding states, together with the Si $s p$ electrons [Fig. 4(b)]. Note that the $4 s \rightarrow 3 d$ electron transfer in the $\mathrm{Mn}_{\text {sub }}$ is not as complete as in the $\mathrm{Mn}_{\text {int }}$, as our calculations find the $\mathrm{Mn}_{\text {sub }} 3 d$ occupation (within the muffin-tin sphere) to be less than the $\mathrm{Mn}_{\text {int }}$ one by 0.2 electrons. Since the nonbonding $e_{\uparrow}$ states (lying below the $t_{2 \uparrow}$ states for $T_{d}$ symmetry) are fully occupied by two electrons, the remaining one electron resides in the $t_{2 \uparrow}$ antibonding band that gives rise to the DOS in the spinup channel in Fig. 3(a).

To conclude, we proposed a stable Si-based heterostructure using $\delta$ doping by interstitial $\mathrm{Mn}$, and proved it to be half-metallic for $1 / 4$ monolayer of Mn, using DFT calculations. A recent STM-DFT study [19] lets us expect that such structures could be prepared by molecular beam epitaxy. In order to achieve ferromagnetic ordering at room temperature, one should aim at higher Mn concentrations. Even for a full $\delta$ layer of $\mathrm{Mn}_{\text {int }}$, the spin polarization of the conduction electrons should still be as high as $60 \%$.

The work at Cologne is supported by the Deutsche Forschungsgemeinschaft through SFB 608.

[1] For a review, see, e.g., I. Žutić, J. Fabian, and S. D. Sarma, Rev. Mod. Phys. 76, 323 (2004).
[2] G. W. Ludwig and H. H. Woodbury, Solid State Phys. 13, 223 (1962).

[3] F. Beeler, O. K. Andersen, and M. Scheffler, Phys. Rev. Lett. 55, 1498 (1985); Phys. Rev. B 41, 1603 (1990).

[4] H. Ohno, Science 281, 951 (1998).

[5] T. Dietl, H. Ohno, F. Matsukura, J. Cibert, and F. Ferrand, Science 287, 1019 (2000).

[6] Y.D. Park, A. T. Hanbicki, S.C. Erwin, C. S. Hellberg, J. M. Sullivan, J. E. Mattson, T. F. Ambrose, A. Wilson, G. Spanos, and B. T. Jonker, Science 295, 651 (2002).

[7] S. Sanvito and N. A. Hill, Phys. Rev. Lett. 87, 267202 (2001).

[8] A. M. Nazmul, T. Amemiya, Y. Shuto, S. Sugahara, and M. Tanaka, Phys. Rev. Lett. 95, 017201 (2005); 96, 149901 (2006).

[9] M. Bolduc, C. Awo-Affouda, A. Stollenwerk, M. B. Huang, F. G. Ramos, G. Agnello, and V.P. LaBella, Phys. Rev. B 71, 033302 (2005).

[10] M.C. Qian, C. Y. Fong, K. Liu, W.E. Pickett, J.E. Pask, and L. H. Yang, Phys. Rev. Lett. 96, 027211 (2006).

[11] S. Picozzi, F. Antoniella, A. Continenza, A. MoscaConte, A. Debernardi, and M. Peressi, Phys. Rev. B 70, 165205 (2004).

[12] A. Stroppa, S. Picozzi, A. Continenza, and A. J. Freeman, Phys. Rev. B 68, 155203 (2003).

[13] T. Dietl, H. Ohno, and F. Matsukura, Phys. Rev. B 63, 195205 (2001).

[14] S.C. Erwin and A. G. Petukhov, Phys. Rev. Lett. 89, 227201 (2002).

[15] G. M. Dalpian, A. J. R. da Silva, and A. Fazzio, Phys. Rev. B 68, 113310 (2003).

[16] X. Luo, S.B. Zhang, and S.H. Wei, Phys. Rev. B 70, 033308 (2004).

[17] A. J. R. da Silva, A. Fazzio, and A. Antonelli, Phys. Rev. B 70, 193205 (2004).

[18] H. Wu, M. Hortamani, P. Kratzer, and M. Scheffler, Phys. Rev. Lett. 92, 237202 (2004).

[19] M. R. Krause, A. J. Stollenwerk, J. Reed, V. P. LaBella, M. Hortamani, P. Kratzer, and M. Scheffler (to be published).

[20] J. P. Perdew, K. Burke, and M. Ernzerhof, Phys. Rev. Lett. 77, 3865 (1996).

[21] P. Blaha et al., WIEN2k (Technical University, Vienna, Austria, 2001).

[22] Structural relaxation breaks the local $T_{d}$ symmetry and splits the $t_{2}$ triplet of the Mn $3 d$ orbitals into $x^{2}-y^{2}, x z$, and $y z$, and the $e$ doublet into $3 z^{2}-r^{2}$ and $x y$ orbitals.

[23] For isolated impurities in $\mathrm{Si}$, the on-site Coulomb correlation could be important. Our GGA $+U$ calculations for $1 / 4 \quad$ ML $\quad \mathrm{Mn} \quad c(4 \times 2)$ with Hubbard $U=3 \mathrm{eV}$ (Refs. [11,24]) and Hund exchange of $0.9 \mathrm{eV}$ show that the half-metallicity remains, whereas it is lost for $U=$ $5 \mathrm{eV}$, but the spin polarization is still higher than $40 \%$. This is also true for the $1 / 4 \mathrm{ML} \mathrm{Mn} p(2 \times 2)$.

[24] A. Ernst, L. M. Sandratskii, M. Bouhassoune, J. Henk, and M. Lüders, Phys. Rev. Lett. 95, 237207 (2005).

[25] E. Şaşığ̆lu, I. Galanakis, L. M. Sandratskii, and P. Bruno, J. Phys. Condens. Matter 17, 3915 (2005).

[26] S. Picozzi, M. Ležaić, and S. Blügel, Phys. Status Solidi A 203, 2738 (2006).

[27] S. A. Centoni, B. Sadigh, G. H. Gilmer, T. J. Lenosky, T. Díaz de la Rubia, and C. B. Musgrave, Phys. Rev. B 72, 195206 (2005). 